\title{
Lipid metabolism offers anticancer treatment by regulating ferroptosis
}

\author{
Michele Carbone ${ }^{1} \cdot$ Gerry Melino $\mathbb{1}^{2,3}$
}

Received: 14 August 2019 / Revised: 29 August 2019 / Accepted: 3 September 2019 / Published online: 23 September 2019

(c) The Author(s), under exclusive licence to ADMC Associazione Differenziamento e Morte Cellulare 2019

Understanding the molecular mechanisms underlying tumour progression is crucial for developing innovative therapies. Initial metabolic changes occur during the process of malignant cell transformation. Subsequently, neoplastic cells adapt their metabolic activities in response to microenvironmental changes occurring throughout cancer progression, from local micro-proliferation to metastasis. Glycolysis, energy production and lipid metabolic adaptation play a crucial role in the interactions that take place between cancer cells and the other cell types present in the microenvironment and between cancer cells and therapeutic drugs. Cancer cell metabolism and the metabolism of the various cell types that form the tumour microenvironment, macrophages, tumour-infiltrating lymphocytes, endothelial cells, and fibroblasts, are all inter-related and thus influence each others activities. Moreover, the local availability of nutrients and oxygen concentration alters the landscape significantly. For example, at lower oxygen pressure, the transcription factor HypoxiaInducible Factor 1 alpha (HIF) is not hydroxylated and degraded in the peroxisome; instead HIF translocates across the nuclear membrane and activates expression of genes that regulated glycolysis, cytotoxicity, transcription, and tumour cell growth. Indeed, while naive or quiescent $\mathrm{T}$ cells meet their bioenergetic needs primarily via the

Michele Carbone

mcarbone@cc.hawaii.edu

$\triangle$ Gerry Melino

gm614@mrc-tox.cam.ac.uk

1 Thoracic Oncology, University of Hawaii Cancer Center, 701 Ilalo St, Honolulu, Hawaii 96813, USA

2 Department of Experimental Medicine, TOR, University of Rome Tor Vergata, 00133 Rome, Italy

3 Medical Research Council, Toxicology Unit, Department of Pathology, Cambridge University, Tennis Court Road, Cambridge CB2 1QP, UK highly efficient ATP-producing mitochondrial oxidative phosphorylation (Kreb's cycle that produces 34 net molecules of ATP), T-cell activation is characterised by a shift in energy production to the less efficient but much more rapid aerobic glycolysis (Warburg effect) that produces only two molecules of ATP, and that over time leads to T-cell exhaustion, and consequent progressive immunologic dysfunction with reduced T-cell proliferation, cytokine production, and diminished antitumor effector capacity. T-cell exhaustion reduces tumour interaction with $\mathrm{T}$ cells (mediated by pMHC/TCR, by LAG3 and by PD-L1/2 and PD-1) and thus the overall ability of immune cells to fight cancer cells. The Tumour Immune MicroEnvironment (TIME) is therefore a highly complex network where metabolism plays a crucial although still partially unexplored, role. Abnormal vascular function, mesenchymal stromal and stem cells, immune-competent cells and lipid-mediated energy, can both modulate hypoxia-restricted drug distribution and its pharmacokinetics. Recent advances on the role of ferroptosis and on the role of lipid metabolism in the TIME, including a better understanding of steroyl CoA desaturase (SCD1) [1] has revealed how lipid metabolism in conjunction with the activation of ferroptosis, may suggest a way to develop novel combinatorial therapeutic approaches using SCD1 inhibitors in combination with ferroptosis inducers.

$\Delta 9$-fatty acyl-CoA desaturase, EC:1.14.19.1, also known as Stearoyl-CoA-desaturase, requires electrons from cytochrome b5 (in the reduced form) as well as $\mathrm{O}_{2}$ in order to insert a double bond into saturated fatty acyl-CoA substrates to produce monounsaturated fatty acids from saturated fatty acids, see Fig. 1a, which in turn are used to produce diacylglycerols, cholesterol esters, wax esters, phospholipids, as well as triglycerides, all major component of cellular membranes. This enzyme, as indicated above and in Fig. 1a, catalyses the formation of a cis double bond in position delta-9 into fatty acyl-CoA substrates including stearoyl-CoA and palmitoyl-CoA and therefore produces both $16: 1$ and 18:1 unsaturated fatty 

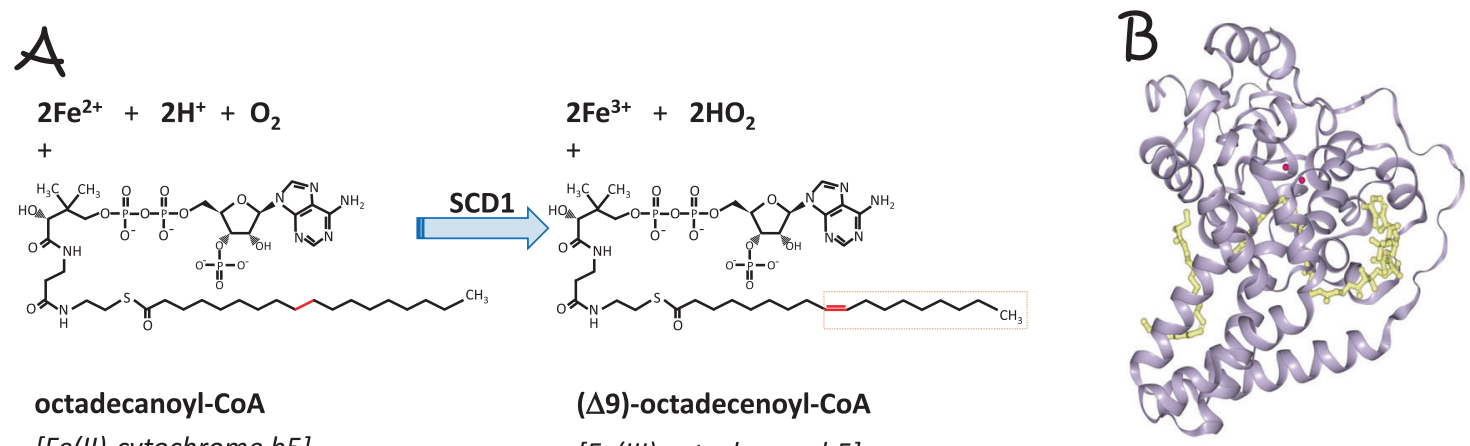

octadecanoyl-CoA

[Fe(II)-cytochrome b5]

$(\Delta 9)$-octadecenoyl-CoA

[Fe(III)-cytochrome b5]

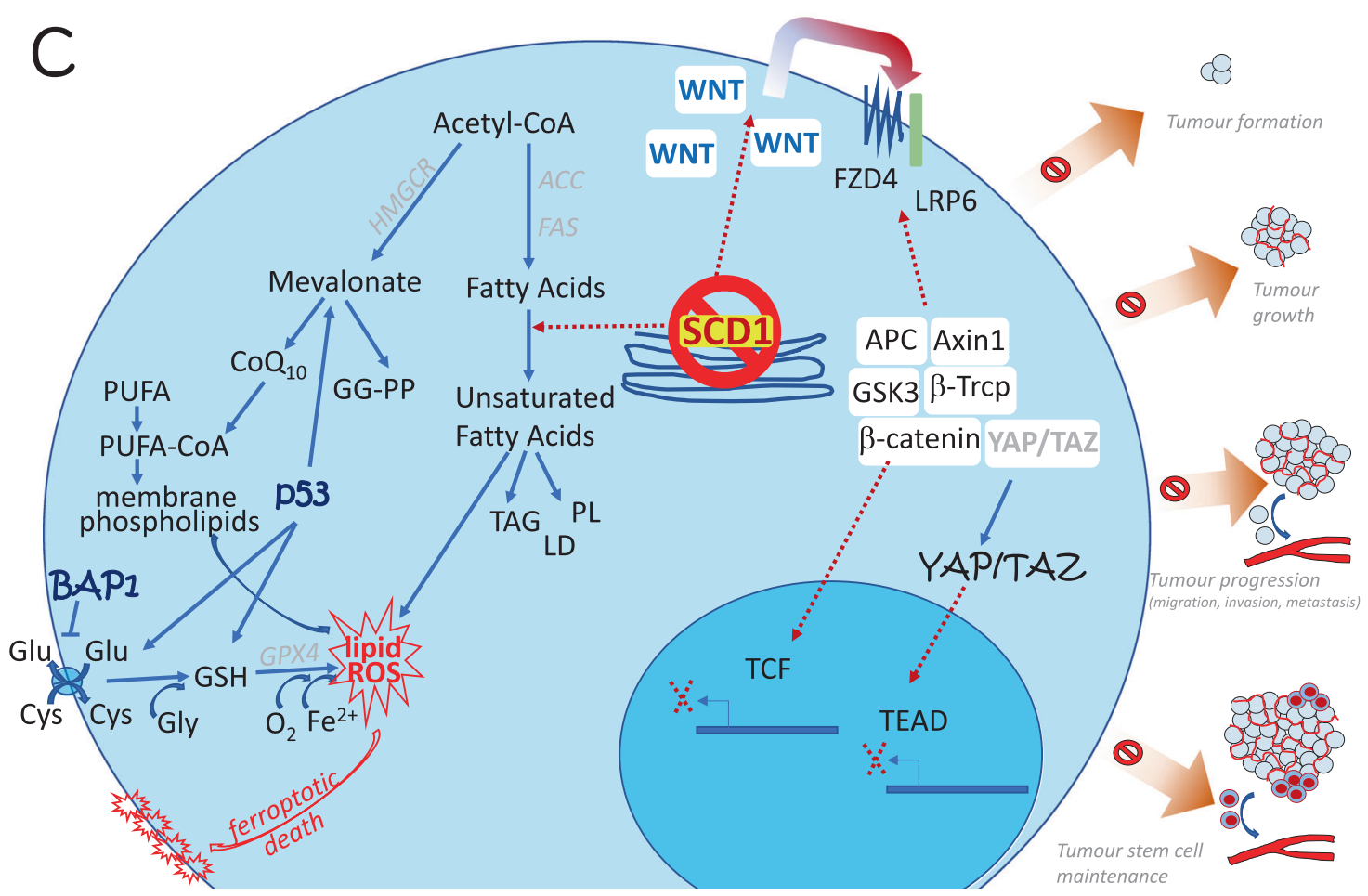

Fig. 1 a Chemical reactions triggered by $\Delta 9$-fatty acyl-CoA desaturase, EC:1.14.19.1, also known as Stearoyl-CoA-desaturases (SCD), introducing a double bond and thus affecting lipid metabolism. b Crystal structure of SCD1. PDB file 4YMK. c Inhibition of SCD1 induces cell death via ferroptosis by impinging on lipid metabolism. Indeed, ferroptosis and SCD1 enzymatic action share a number of lipid mediators, linking the two phenomena. At the same time it acts on cancer stem cells by regulating the autocrine Wnt pathway and the hippo pathway, shown on the intracellular right side, as well as by regulating apoptotic programmed cell death, not shown. Both actions contribute to the regulation of different stages of cancer progression, as highlighted on the far right. See main text for details. ACC acetyl-CoA carboxylase, FAS fatty acids synthase, GGPP geranylgeranyl pyrophosphate, GG-transferase geranylgeranyl transferase, HMGCR HMG CoA-reductase, PL phospholipids, TAG triacylglycerols, YAP Yes associated protein, TAZ transcriptional co-activator with PDZ binding motif (tafazzin), TEAD TEA domain transcription factor 1 , GSH glutathione, PE phosphatidylethanolamine, PUFA polyunsaturated fatty acyl chains, GPX4 glutathione peroxidase 4, ROS reactive oxygen species, SCD1 steroyl CoA desaturase 1 acids. SCD, Fig. 1b, is a crucial enzyme in lipid metabolism as replicating cells must synthetize fatty acids for energetic, structural, and signalling purposes. Two human isoforms are present: SCD5, is localised in pancreas and brain, while SCD1 is ubiquitous. SCD1 consistently inhibits AMPK, enhances Akt and lipid metabolism in general by enhancing lipogenesis and lowering fatty acid oxidation; in other words, it promotes cancer progression. Conversely, SCD1 inhibition induces programmed cell death by apoptosis [2], and inhibits cancer stem cells [3]. As shown in Fig. 1c, SCD1 regulates the autocrine action of the wnt/ $\beta$-catenin cascade on fatty acid metabolism, modulating the YAP/TAZ pathway in lung cancer stem cells. Accordingly, SCD1 inhibitors modulate distinct 
stages of tumour promotion and progression, including the EGF, ER stress, NF-kB, and PI3K/AKT/HIF2 pathways [4]. Very recently the team of S. Torti [1], demonstrated that SCD1 is highly expressed in ovarian cancer cells, where SCD1 protects ovarian cancer cells from programmed cell death. Accordingly, SCD1 direct products, palmitoleic acid, oleate, palmitoyl CoA and stearolyl CoA C16:1 and C18:1 show the same biological effects, while SCD1 inhibition at pharmaceutical (using MF-438 or CAY10566) or genetic (knockdown) levels triggers cell death, both by apoptosis (zVAD-fmk inhibitable) and ferroptosis (FER-1 inhibitable). Interestingly, ferroptosis and SCD1 metabolism share common lipid mediators $[5,6]$. SCD1 inhibition triggers ferroptosis with reduced synthesis of cytoprotective lipids, including reduction of the mevalonate metabolite $\mathrm{CoQ}_{10}$. These results have been validated in an ovarian cancer mouse model [1].

Among several genes capable of modulating different aspects of cell metabolism, the TP53 and BAP1 genes, stand apart, because of their powerful tumour suppressor activity: all individuals born with inherited heterozygous TP53 mutations (Li-Fraumeni cancer syndrome) and BAP1 heterozygous mutations (BAP1 cancer syndrome) [7] develop one or more malignancies during their lifetime. Both TP53 and BAP1 are able to simultaneously regulate DNA repair by homologous recombination, cell death (both apoptosis and ferropoptosis), and mitochondrial metabolism. These powerful activities may explain why mutations of these two genes are so significcant at inducing cancer, compared with mutations in other tumour suppressors that regulate only some of these three crucial pathways. We suggest that the status of p53 and BAP1 (wild type versus mutated) may influence the therapeutic efficacy of ferroptotic-based therapies in cancer [8]. Indeed, several of the pathways outlined in Fig. 1 are regulated by TP53 [9-14] and BAP1 [7].

In conclusion, there are several reasons to consider SCD1 a powerful drug target in cancer: it triggers (i) apoptosis, (ii) ferroptosis, and (iii) the hippo pathway and wnt/ $\beta$-catenin action, see Fig. 1c. Both SCD1 and ferroptosis are druggable, offering new potential combined therapeutic intervention, as elegantly shown by Torti's team [1]. Selectivity of inhibition of SCD1 has been achieved using nicotinamide, pyridazine or piperazinylpyridine derivatives, including BZ36, SW203668, CVT-12012, SSI-4, MF-438, and A939572 [4]. More selective and less toxic compounds should be studied, but more work is required to define which tumour should be targeted and how patients should be selected and stratified to properly tailor individual patient needs [15]. The fact that SCD1 inhibition can trigger ferroptosis expands the choice of combined therapies currently available in cancer.
Funding MC grants: National Institutes of Health/National Cancer Institute, the US Department of Defense, and the UH Foundation ("Pathogenesis of Malignant Mesothelioma"; Honeywell International Inc, Riviera United-4-a Cure, Maurice and Joanna Sullivan Family Foundation); has a patent pending application for BAP1, a patent issued for "Using Anti-HMGB1 Monoclonal Antibody or other HMGB1 Antibodies as a Novel Mesothelioma Therapeutic Strategy," and a patent issued for "HMGB1 As a Biomarker for Asbestos Exposure and Mesothelioma Early Detection"; and is a board-certified pathologist who provides consultation for mesothelioma expertise and diagnosis, including paid medical-legal consulting. GM grants: UK Medical Research Council, Associazione Italiana per la Ricerca contro il Cancro (AIRC) to GM IG\#20473 (2018-2022), Ministry of Health \& MAECI Italy-China Science and Technology Cooperation (\#PGR00961), and Regione Lazio through Lazio Innova Progetto Gruppo di Ricerca n 85-2017-14986; consults and works for Springer Nature.

\section{Compliance with ethical standards}

Conflict of interest The authors declare that they have no conflict of interest.

Publisher's note Springer Nature remains neutral with regard to jurisdictional claims in published maps and institutional affiliations.

\section{References}

1. Tesfay L, Paul BT, Konstorum A, Deng Z, Cox AO, Lee J, et al. Steroyl-CoA Desaturase 1 (SCD1) protects ovarian cancer cells from ferroptotic cell death. Cancer Res. 2019. https://doi. org/10.1158/0008-5472.CAN-19-0369. [Epub ahead of print]

2. Pisanu ME, Noto A, De Vitis C, Morrone S, Scognamiglio G, Botti G, et al. Blockade of Stearoyl-CoA-desaturase 1 activity reverts resistance to cisplatin in lung cancer stem cells. Cancer Lett. 2017;406:93-104. https://doi.org/10.1016/j.canlet.2017. 07.027

3. Noto A, De Vitis C, Pisanu ME, Roscilli G, Ricci G, Catizone A, et al. Stearoyl-CoA-desaturase 1 regulates lung cancer stemness via stabilization and nuclear localization of YAP/TAZ. Oncogene. 2017;36:4671-2. https://doi.org/10.1038/onc.2017.212

4. Tracz-Gaszewska Z, Dobrzyn P. Stearoyl-CoA desaturase 1 as a therapeutic target for the treatment of cancer. Cancers (Basel). 2019;11:E948. https://doi.org/10.3390/cancers11070948

5. Dixon SJ, Stockwell BR. The hallmarks of ferroptosis. Annu Rev Cancer Biol. 2019;3:35-54.

6. Hassannia B, Vandenabeele P, Vanden Berghe T. Targeting ferroptosis to iron out cancer. Cancer Cell 2019;35:830-49. https:// doi.org/10.1016/j.ccell.2019.04.002

7. Carbone M, Adusumilli PS, Richard Alexander Jr H, Baas P, Bardelli F, Bononi A, et al. Mesothelioma scientific clues fro prevention, diagnosis, and therapy. CA Cancer J Clin. 2019;69:402-29. https://doi.org/10.3322/caac.21572

8. Carbone M, Melino G. Steroyl CoA desaturase regulates ferroptosis in ovarian cancer offering new therapeutic perspectives. Cancer Res. 2019. In press.

9. Affar EB, Carbone M. BAP1 regulates different mechanisms of cell death. Cell Death Dis. 2018;9:1151. https://doi.org/10.1038/ s41419-018-1206-5

10. Furth N, Aylon Y. The LATS1 and LATS2 tumor suppressors: beyond the Hippo pathway. Cell Death Differ. 2017;24:1488-501. https://doi.org/10.1038/cdd.2017.99 
11. Levine AJ. Reviewing the future of the P53 field. Cell Death Differ. 2018;25:1-2. https://doi.org/10.1038/cdd.2017.181

12. Jiang L, Kon N, Li T, Wang SJ, Su T, Hibshoosh H, et al. Ferroptosis as a p53-mediated activity during tumour suppression. Nature. 2015;520:57-62.

13. Aubrey BJ, Kelly GL, Janic A, Herold MJ, Strasser A. How does p53 induce apoptosis and how does this relate to p53-mediated tumour suppression? Cell Death Differ. 2018;25:104-13. https:// doi.org/10.1038/cdd.2017.169
14. Sullivan KD, Galbraith MD, Andrysik Z, Espinosa JM. Mechanisms of transcriptional regulation by p53. Cell Death Differ. 2018;25:133-43. https://doi.org/10.1038/cdd. 2017.174

15. Carbone M, Amelio I, Affar EB, Brugarolas J, Cannon-Albright LA, Cantley LC, et al. Consensus report of the 8 and 9th Weinman Symposia on Gene x Environment Interaction in carcinogenesis: novel opportunities for precision medicine. Cell Death Differ. 2018;25:1885-904. 